\title{
Synchrony effects in automatic and controlled retrieval
}

\author{
LIXIA YANG \\ University of Toronto, Toronto, Ontario, Canada \\ LYNN HASHER \\ University of Toronto, Toronto, Ontario, Canada \\ and Rotman Research Institute of Baycrest Center, Toronto, Ontario, Canada \\ AND \\ DARYL E. WILSON \\ University of Toronto, Toronto, Ontario, Canada
}

\begin{abstract}
Using a speeded retrieval procedure, we investigated time-of-day effects in automatic and controlled retrieval. Morning-type adults were tested at either peak (early morning) or off-peak (late afternoon) times on a speeded implicit (Experiment 1) or explicit (Experiment 2) stem completion task. In Experiment 1, retrieval strategies were identified by changes in response speed between a practice phase with rapid retrieval and an implicit memory test phase. Performance based on controlled retrieval (shown by slowdown participants) showed more priming at peak than at off-peak times of day, a finding confirmed in Experiment 2, in which the participants were given intentional retrieval instructions when the materials switched. In contrast, performance based on automatic retrieval (shown by nonslowdown participants) did not differ across peak and off-peak times. The finding suggests a robust synchrony effect in controlled retrieval, but not in automatic retrieval, which does not appear to vary across the day.
\end{abstract}

Performance on explicit memory tasks is widely reported to be better at peak than at off-peak times of day for both younger and older adults (e.g., Hasher, Chung, May, \& Foong, 2002; Hasher, Goldstein, \& May, 2005; Petros, Beckwith, \& Anderson, 1990; West, Murphy, Armilio, Craik, \& Stuss, 2002; Yoon, 1997). ${ }^{1}$ Similar synchrony effects have been reported, again for both younger and older adults, in tasks requiring controlled processing, including regulation of distraction (May, 1999) and regulation of strong responses (May \& Hasher, 1998), as well as on a measure of adolescents' fluid intelligence (Goldstein, Hahn, Hasher, Wiprzycka, \& Zelazo, 2007).

In contrast with this emerging literature examining controlled processes across time of day, there is little evidence regarding synchrony effects on automatic processes. To our knowledge, there is only one such study, and it reported a reverse synchrony effect for implicit, as compared with explicit, memory performance; that is, both younger and older adults showed better performance at their off-peak than at their peak times of day (May, Hasher, $\&$ Foong, 2005).

In this study, we sought to do a conceptual replication of the dramatic dissociation in the synchrony effects in explicit and implicit memory. In selecting a task, we took account of evidence that implicit performance can be either facilitated (e.g., Jacoby, 1991) or disrupted (e.g., Beilock, Bertenthal, McCoy, \& Carr, 2004; Howard \& Howard, 2001; Roßnagel, 2001) by intentional processes, presumably depending on whether the intentional process is congruent or incongruent with the automatic process. In the present study, we chose a procedure with an intention to minimize reliance on intentional processes by training participants to retrieve words very rapidly prior to introducing an implicit test of memory (Horton, Wilson, \& Evans, 2001; Wilson \& Horton, 2002). A unique advantage of this procedure is that it enables us to discriminate between individuals who likely use controlled retrieval and those who rely primarily on automatic retrieval by tracking changes in their retrieval speed.

In this procedure, participants are initially trained to use cues to retrieve words rapidly from semantic memory before an implicit test is introduced that consists of a mix of cues, some solvable only with words from semantic memory and some solvable with words presented in an earlier study phase. Individual retrieval strategies can be determined by comparing each participant's response times in the semantic retrieval phase with those in the critical test phase. Given evidence that controlled retrieval takes lon-

L. Yang or L. Hasher, lixiay@ryerson.ca or hasher@psych.utoronto.ca 
ger than automatic retrieval (Richardson-Klavehn \& Gardiner, 1995), we assume that participants who slow down from the semantic retrieval phase to the test phase are likely switching to controlled retrieval strategies, whereas those who continue to respond rapidly are relying on automatic retrieval. Having identified those participants who continue to rely on automatic processes and those who switch to controlled processes, we then compare priming scores across time of day separately for these two groups to examine the synchrony effects on the two different retrieval processes.

We report two experiments in which this procedure was used. In Experiment 1, participants were uninformed about the change in cues between the retrieval training phase (with its cues to new words) and the testing phase (with its mix of cues to old and new words), and we examined time-of-day effects for the participants who relied on more automatic versus more controlled retrieval. In Experiment 2 , we explicitly instructed the participants to use controlled retrieval to validate the time-of-day effects seen in Experiment 1 for those participants identified as using controlled retrieval. To date, there is substantial and consistent evidence that younger and older adults show identical synchrony effects (e.g., Hasher et al., 2005; Hasher, Zacks, \& May, 1999), despite an overall age-related decline in episodic memory (e.g., Balota, Dolan, \& Duchek, 2000). Thus, although there are limits to generalizability, we tested only morning-type older adults.

\section{EXPERIMENT 1}

There were three major phases in the experiment: study, retrieval practice, and test. In the study phase, the participants completed stems (e.g., ELE__ ), each in response to a semantic cue (e.g., large animal with a trunk). In the retrieval practice phase, the participants were trained to quickly generate words to novel stems. In the test phase, speed of generation continued to be encouraged, but now some stems could be completed by words generated during the study phase. The participants were not informed about the change in materials.

\section{Method}

\section{Participants}

The final sample consisted of 53 healthy morning-type older adults (ages, 60-76 years; $M=67.89, S D=4.19$ ). They were screened using the Morningness-Eveningness Questionnaire (MEQ; Horne \& Ostberg, 1976), a valid and reliable tool for determining temporal preference patterns (e.g., Smith, Reilly, \& Midkiff, 1989). Their mean MEQ score was 67.32 (range: 59-80), within the range of morning-type people (59-86; Horne \& Ostberg, 1976). ${ }^{2}$ The Short Blessed Test (SBT; Pfeiffer, 1975) was used to screen for cognitive impairment. Twenty-seven participants were tested in the morning (9-10 a.m.) and 26 in the afternoon (4-5 p.m.). These testing times and cutoff scores were similar to those used in previous work, including May et al.'s study (2005). Each participant received 10 Canadian dollars as compensation. Nine original participants were replaced, 1 for being a nonnative English speaker, 1 for having a high SBT score, 2 due to technical problems, and 5 for reporting the intentional use of studied words to complete the test stems. The morning-testing and afternoon-testing groups did not differ on the demographic variables displayed in Table $1(t \mathrm{~s}<1.34, p \mathrm{~s}>.18)$.
Table 1

Demographic Characteristics of the Final Sample

\begin{tabular}{|c|c|c|c|c|c|}
\hline & \multirow[b]{3}{*}{ Variables } & \multicolumn{4}{|c|}{ Testing Time } \\
\hline & & \multicolumn{2}{|c|}{ Morning } & \multicolumn{2}{|c|}{ Afternoon } \\
\hline & & $M$ & $S D$ & $M$ & $S D$ \\
\hline \multirow[t]{5}{*}{ Experiment 1} & Age & 68.56 & 4.41 & 67.19 & 3.91 \\
\hline & Years of education & 16.63 & 3.19 & 16.19 & 2.33 \\
\hline & Vocabulary & 33.74 & 8.70 & 34.55 & 8.24 \\
\hline & MEQ & 67.81 & 5.27 & 66.81 & 5.39 \\
\hline & SBT & 0.26 & 0.66 & 0.54 & 0.86 \\
\hline \multirow[t]{5}{*}{ Experiment 2} & Age & 65.95 & 4.98 & 66.50 & 4.13 \\
\hline & Years of education & 15.59 & 2.56 & 16.38 & 3.23 \\
\hline & Vocabulary & 27.50 & 9.23 & 31.62 & 8.32 \\
\hline & MEQ & 65.98 & 4.27 & 64.83 & 3.81 \\
\hline & SBT & 0.27 & 0.70 & 0.71 & 1.40 \\
\hline
\end{tabular}

Note-In Experiment 1, $n=27$ for the morning-testing group and $n=$ 26 for the afternoon-testing group; in Experiment 2, $n=22$ for the morning-testing group and $n=24$ for the afternoon-testing group. Vocabulary was measured with an Extended Range Vocabulary Test (Educational Testing Service, 1976). MEQ, Morningness-Eveningness Questionnaire (Horne \& Ostberg, 1976); SBT, Short Blessed Test (Pfieffer, 1975).

\section{Materials}

Four lists of critical words, each with 24 words, one list of 24 fillers, and eight buffers, were selected from the items used by Wilson and Horton (2002). No proper nouns were used, and all the words had straightforward spellings and unique three-letter stems. The stems for the four lists of critical items had an equal average baseline completion rate of .23 in Wilson and Horton (2002), as well as in the context of the present study. The lists were counterbalanced across participants and testing times (i.e., morning vs. afternoon) so that each list served equally often as a study list, as a nonstudy control list, and as one of the two practice lists. In the study phase, the 24 critical words were presented intermixed with 24 filler words, with 2 buffer items at the beginning and 2 others at the end of the list. Fillers were used to reduce awareness of the connection between the study and the test lists. In order to track changes in retrieval speed and also to encourage rapid responding, the retrieval practice phase was divided into two phases, each with 24 items from one of the two practice lists. For the same purpose, the final test phase was also divided into two phases, each of which started with 4 buffer items followed by 24 stems, 12 from the study list (i.e., old stems) and 12 from the nonstudy control list (i.e., new stems). The buffer items in both the study and the test phases served to eliminate warm-up effects on response times for critical items.

\section{Procedure}

Figure 1 illustrates the basic experimental procedure in Experiments 1 and 2 .

Study phase. The participants generated a series of words, each in response to a semantic cue along with a three-letter stem, both of which were presented on a computer screen until a response was made.

Filler task phase. A 10-min number fragment completion task required the participants to complete a series of equations (e.g., for " 2 _ $+15=35$," answer " 0 "). The filler task prevented the participants from deliberately rehearsing the studied items.

Retrieval practice and test phases. The participants were instructed to quickly complete a series of word stems with the first word that came to mind. Both the practice and the test phases were further divided into two in order to put pressure on retrieval speed by providing mean response times at the end of each phase, along with instructions urging the participants to try to go faster in the next phase. Subdividing the practice and test phases into two for each also enabled us to closely track speed changes across the test. Note that the instructions did not differ for the practice and the test phases; thus, to the participants, the test phase seemed to be a continuation of the practice phase. 


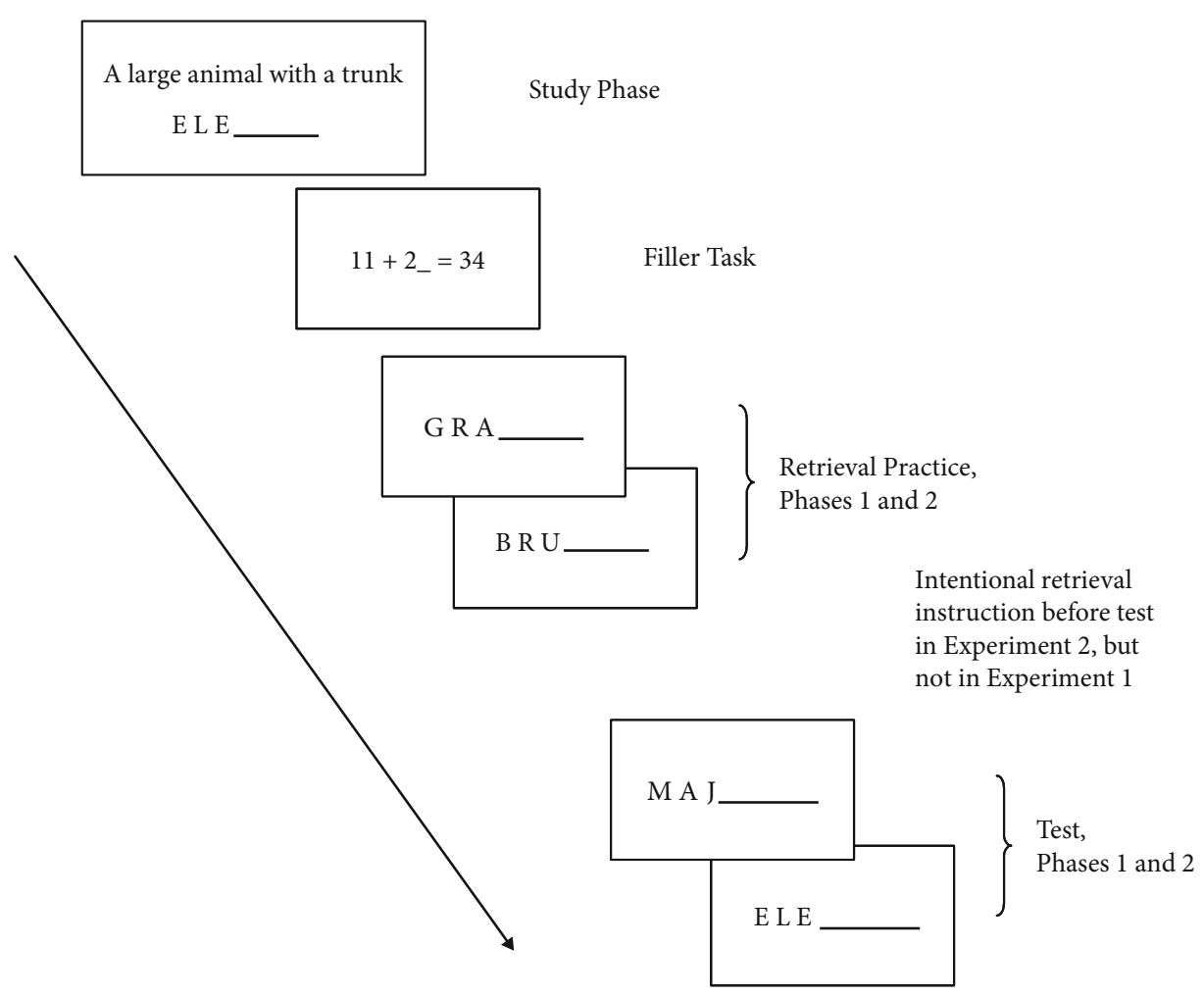

Figure 1. Experimental procedure in Experiments 1 and 2.

Finally, the participants were given an awareness questionnaire, a background questionnaire, the Extended Range Vocabulary Test (Educational Testing Service, 1976), and the SBT.

\section{Results}

\section{Response Times}

For each participant, we calculated median response times (RTs) for the 24 items in the first and second retrieval practice phases, as well as for the 12 new (i.e., nonstudied) items in each of the two test phases (see Figure 2). The data from one outlier ( $>3 S D$ s) in the practice phase were already excluded in the final sample. An ANOVA with time of testing as a between-subjects factor and test phase as a within-subjects factor revealed only a reliable phase effect $\left[F(3,153)=23.34, M S_{\mathrm{e}}=11,003.57, p<\right.$ $.001]$, stemming from the speedup from the first to the second retrieval practice phase $[t(52)=5.47, p<.001]$; speed did not change thereafter $(t \mathrm{~s}<1.06)$.

Because our main interest was in priming at peak and off-peak times of day by participants who employed different retrieval strategies, we divided the morning-tested and afternoon-tested participants into two groups on the basis of the individuals' retrieval speed change from the second retrieval practice phase to the combined test phases. Twenty-five participants ( 14 tested in the morning and 11 in the afternoon) slowed down (i.e., $\mathrm{RT}_{\text {test }}-\mathrm{RT}_{\text {practice } 2}>0$ ) and, thus, were presumably using controlled retrieval. The average slowdown of this group was $62.7 \mathrm{msec}[S D=$ $55.9 ; t(24)=5.62, p<.001]$. Twenty-eight participants
(13 tested in the morning and 15 in the afternoon) did not slow down (i.e., $\mathrm{RT}_{\text {test }}-\mathrm{RT}_{\text {practice } 2}<0$ ) and, thus, were presumed to be relying on automatic retrieval. In fact, on average, this nonslowdown group actually sped up by $71.0 \mathrm{msec}[S D=60.7 ; t(27)=6.20, p<.001]$. Note that the two groups produced by this RT split did not differ in their demographic characteristics $\left(F_{\mathrm{s}}<2.06, p \mathrm{~s}>.15\right)$.

\section{Priming Effects}

To assess retrieval, we calculated a priming score by subtracting the baseline stem completion rate (i.e., the percentage of control stems completed with nonstudied critical words) from the target completion rate (i.e., the percentage of studied stems completed with studied words). An overall 2 (testing time: morning vs. afternoon) $\times 2$ (retrieval strategy: controlled vs. automatic) betweensubjects ANOVA on the priming score revealed greater priming in the morning $(M=27.62, S D=12.51)$ than in the afternoon $(M=19.71, S D=14.32)[F(1,49)=5.17$, $\left.M S_{\mathrm{e}}=172.81, p<.05\right]$. This main effect was accompanied by a reliable interaction between testing time and retrieval strategy $\left[F(1,49)=4.11, M S_{\mathrm{e}}=172.81, p<\right.$ $.05]$. Planned contrasts indicated that the overall timeof-day effect was driven solely by the group engaged in controlled retrieval. They showed greater priming in the morning $(M=31.55, S D=11.75)$ than in the afternoon $(M=15.91, S D=16.96)[t(23)=2.72, p<.05]$. In contrast, the automatic retrieval group did not differ in priming across time of day [morning, $M=23.40, S D=12.33$; 


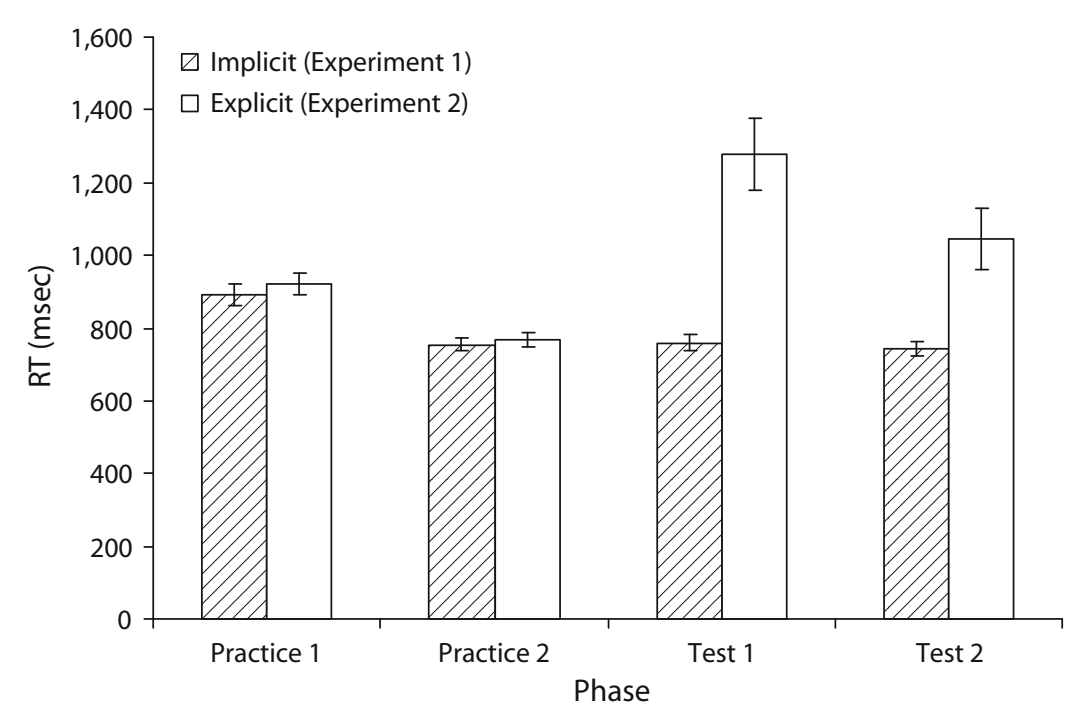

Figure 2. Median response times (RTs) as a function of phase and task. Error bars refer to the mean standard errors.

afternoon, $M=22.50, S D=11.87 ; t(26)=0.20, p=$ .85 ; see the two panels on the left in Figure 3].

The data suggest that automatic retrieval, indexed by the performance of the participants who maintained a rapid speed in the test phase, was invariant across the day. By contrast, controlled retrieval, indexed by the performance of the participants who slowed down in the test phase, was better at peak than at off-peak times. To verify the suggestion that a slowdown in responding is driven primarily by engaging in controlled retrieval, we conducted Experiment 2, using an explicit version of the present task.

\section{EXPERIMENT 2}

The only change from the first experiment was that the participants were fully informed, prior to the test phase, that some of the stems could be completed with initially studied words. Furthermore, the participants were encouraged to use those studied words to complete the stems. If the slowdown in Experiment 1 was, as we suggest, driven primarily by the use of controlled retrieval, we should see a slowdown in Experiment 2 when the participants were instructed to switch to controlled retrieval, along with greater priming at a peak than at an off-peak time of day.

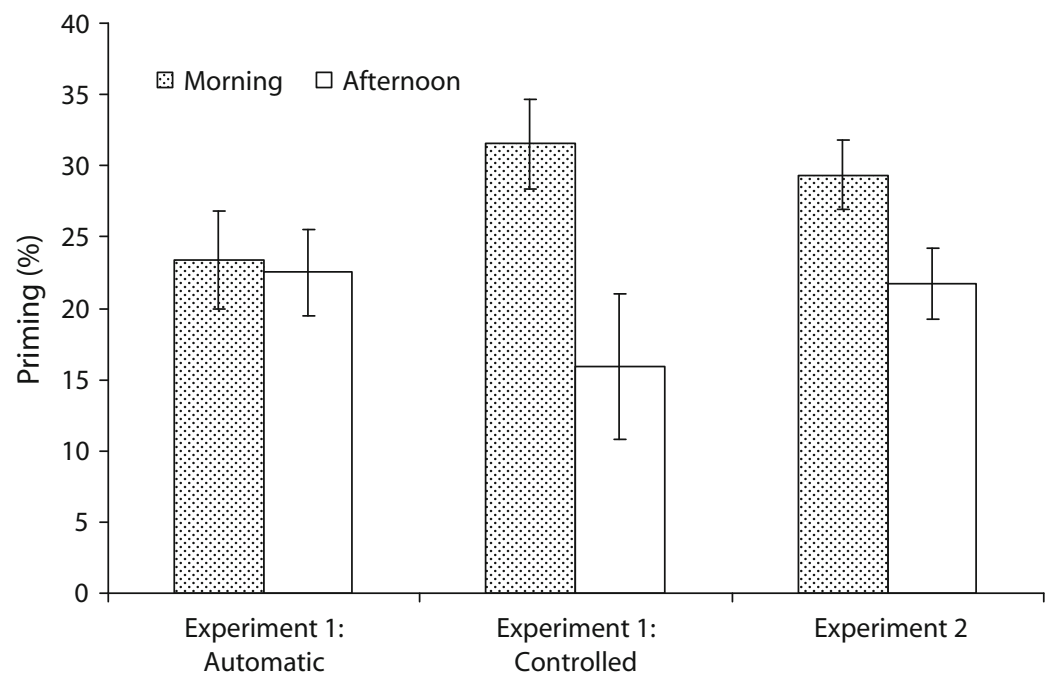

Figure 3. The percentage of priming as a function of testing time, with the leftmost panel for the group using automatic retrieval in the implicit memory task (Experiment 1), the middle panel for the group engaging in controlled retrieval in the implicit memory task (Experiment 1), and the rightmost panel for the explicit memory task (Experiment 2). Error bars refer to the mean standard errors. 


\section{Method}

\section{Participants}

The final sample consisted of 46 healthy older adults (ages, 60-78 years; $M=66.24, S D=4.51$ ), with a mean MEQ score of 65.38 (range: 60-74), within the range of morning-type (59-86; Horne \& Ostberg, 1976). All the participants scored below a cutoff score of 6 on the SBT. Twenty-two participants were tested in the morning (910 a.m.), and 24 were tested late in the afternoon (4-5 p.m.). Six of the original participants were replaced, 2 for being nonnative English speakers and 4 due to health issues or technical problems. The morning-testing and afternoon-testing groups did not differ on any of the demographic variables (see Table $1 ; t \mathrm{~s}<1.59, p \mathrm{~s}>.11$ ).

\section{Results}

\section{Response Times}

An ANOVA revealed only a reliable main effect of phase $\left[F(3,132)=15.97, M S_{\mathrm{e}}=136,827.73, p<.001\right.$; see Figure 2]. As in the first experiment, there was a reliable speedup in responding from the first to the second retrieval practice phase $[t(45)=7.36, p<.001]$. As was anticipated, there was a reliable slowdown between the second practice retrieval phase and the collapsed test phases $[t(45)=4.15, p<.001]$. An inspection of the individual participants' data, using the same standard as that in Experiment 1, confirmed that 43 out of 46 participants slowed down, consistent with the intentional retrieval instructions. The pattern of results for both RTs and priming scores remained unchanged after the scores for the 3 participants who had not slowed down were removed. Note that there was a speedup between the first and second test phases [see Figure 2; $t(45)=3.94, p<.01$ ]. This speedup within test might be due to a practice effect, to a decreased reliance on controlled retrieval, or to both, but it is not consistent with a suggestion that people slow down in this task because they are fatigued.

\section{Priming}

As before, priming scores were calculated by subtracting the baseline stem completion rate from the target completion rate. An analysis of the priming scores showed greater priming in the morning $(M=29.36, S D=11.54)$ than in the afternoon $(M=21.70, S D=12.04)[t(44)=$ 2.20, $p<.05$; see the rightmost panel in Figure 3]. This pattern parallels that seen for the participants in the first experiment who slowed down during the test phase, and is consistent with the results of a number of explicit memory tasks (Petros et al., 1990; West et al., 2002; Yoon, 1997).

\section{GENERAL DISCUSSION}

The results of these two experiments suggest that controlled retrieval is better at a peak than at an off-peak time, whereas truly automatic retrieval appears to be unaffected by time of day. The synchrony effect observed here for controlled retrieval replicates a robust effect in the literature (e.g., Anderson, Petros, Beckwith, Mitchell, \& Fritz, 1991; Hasher et al., 2005; May \& Hasher, 1998; May et al., 2005; Petros et al., 1990; West et al., 2002; Winocur \& Hasher, 2002; Yoon, 1997). We found the synchrony effect both when the participants chose controlled retrieval in an ostensibly implicit memory task (i.e., the participants who slowed down during test in Experiment 1) and when the participants were instructed to use controlled retrieval (Experiment 2).

In contrast to the synchrony effect for controlled retrieval, we found no difference in priming across the two testing times for the participants who relied on automatic retrieval (i.e., those who did not slow down in Experiment 1), suggesting the possibility that automatic retrieval is invariant across the day. At first glance, this finding is at odds with May et al.'s (2005) evidence of a reverse synchrony effect for implicit memory - that is, better performance at off-peak that at peak times of day. Although the precise source of this difference remains to be determined, we offer a speculation based on the cover story used by May et al., which described the implicit test as a game in which word knowledge was to be assessed and in which no time limit was given for generating a word. At peak times, when the participants were most alert and proactive, they might have occasionally rejected the first, automatically generated word in favor of producing a word they judged to better reflect their knowledge. As a consequence, output from automatic retrieval may have been masked, resulting in an underestimation of automatic priming at peak times. In support of this argument, we note that providing participants too much time can interfere with their implicit performance (Roßnagel, 2001), as can providing participants with instructions to search for patterns in implicit learning (Howard \& Howard, 2001). Similar evidence for the blocking or masking of automatic processes by deliberate processes is also reported in the skill-learning literature (Beilock et al., 2004).

Our findings have implications for the design of educational programs and the organization of daily activities. Effortful or controlled activities or programs will clearly be most efficient when scheduled at an individual's peak time of day. Because there is considerable evidence of age and individual differences in peak times of day, beginning at least prior to adolescence and extending through the life span (Hasher et al., 2005; Kim, Dueker, Hasher, \& Goldstein, 2002; Roenneberg et al., 2004), taking these into account may optimize school achievement and work success for a majority of individuals.

Automatic processes may not change across the day, suggesting the possibility that pure automatic processes can be effective even at off-peak times of day. Recent evidence suggests that even difficult tasks, such as decision making, can rely on automatic processes (e.g., Dijksterhuis \& Nordgren, 2006) and, so, may be successfully performed at off-peak times of day. By contrast, if automatic processes are vulnerable to strategic facilitation (e.g., Jacoby, 1991) in some circumstances and to disruption (e.g., Beilock et al., 2004; Howard \& Howard, 2001; May et al., 2005; Roßnagel, 2001) in others, performance at peak times will be complexly determined. The untangling of facilitation and interference effects from deliberate processes to automatic ones is a matter for further research. In closing, we acknowledge that although all studies to date have reported synchrony effects that are comparable 
for both older and younger adults, the present time-of-day conclusions are based on a sample of older adults, and so, there may be limits to their generalizability.

\section{AUTHOR NOTE}

This work was supported by Grant R37 AGO4306 from the National Institute on Aging. We thank Carol Wong, Rachelle Ta-Min, Ji-A Min, and Ursula Wiprzycka for their assistance. Correspondence concerning this article should be addressed to L. Yang, Department of Psychology, Ryerson University, 350 Victoria Street, Toronto, ON, M5B 2K3 Canada or to L. Hasher, Department of Psychology, University of Toronto, 100 St. George Street, Toronto, ON, M5S 3G3 Canada (e-mail: lixiay@ ryerson.ca or hasher@psych.utoronto.ca).

\section{REFERENCES}

Anderson, M. J., Petros, T. V., Beckwith, B. E., Mitchell, W. W., \& FRITZ, S. (1991). Individual differences in the effect of time of day on long-term memory access. American Journal of Psychology, 104, 241-255.

Balota, D. A., Dolan, P. O., \& Duchek, J. M. (2000). Memory changes in healthy older adults. In E. Tulving \& F. I. M. Craik (Eds.), The Oxford handbook of memory (pp. 395-409). New York: Oxford University Press.

Beilock, S. L., Bertenthal, B. I., McCoy, A. M., \& Carr, T. H. (2004). Haste does not always make waste: Expertise, direction of attention, and speed versus accuracy in performing sensorimotor skills. Psychonomic Bulletin \& Review, 11, 373-379.

Dijksterhuis, A., \& Nordgren, L. F. (2006). A theory of unconscious thought. Perspectives on Psychological Science, 1, 95-109.

Educational Testing Service (1976). Kit of factor-referenced cognitive tests. Princeton, NJ: Author.

Goldstein, D., Hahn, C., Hasher, L., Wiprzycka, U. J., \& Zelazo, P. D. (2006). Time of day, intellectual performance, and behavioral problems in morning versus evening type adolescents: Is there a synchrony effect? Personality \& Individual Differences, 42, 431-440.

Hasher, L., Chung, C., May, C. P., \& Foong, N. (2002). Age, time of testing, and proactive interference. Canadian Journal of Experimental Psychology, 56, 200-207.

Hasher, L., Goldstein, D., \& May, C. (2005). It's about time: Circadian rhythms, memory, and aging. In C. Izawa \& N. Ohta (Eds.), Human learning and memory: Advances in theory and application. The 4th Tsukuba International Conference on Memory (pp. 199-217). Mahwah, NJ: Erlbaum.

HASHER, L., ZACKS, R. T., \& MAY, C. P. (1999). Inhibitory control, circadian arousal, and age. In D. Gopher \& A. Koriat (Eds.). Attention and performance XVII: Cognitive regulation of performance. Interaction of theory and application (pp. 653-675). Cambridge, MA: MIT Press.

Horne, J., \& Ostberg, O. (1976). A self-assessment questionnaire to determine morningness-eveningness in human circadian rhythms. International Journal of Chronobiology, 4, 97-110.

Horton, K. D., Wilson, D. E., \& Evans, M. (2001). Measuring automatic retrieval. Journal of Experimental Psychology: Learning, Memory, \& Cognition, 27, 958-966.

Howard, D. V., \& Howard, J. H., JR. (2001). When it does hurt to try: Adult age differences in the effects of instructions on implicit pattern learning. Psychonomic Bulletin \& Review, 8, 798-805.

JACOBY, L. L. (1991). A process dissociation framework: Separating automatic from intentional uses of memory. Journal of Memory \& Language, 30, 513-541.
Kim, S., Dueker, G. L., Hasher, L., \& Goldstein, D. (2002) Children's time of day preference: Age, gender and ethnic differences. Personality \& Individual Differences, 33, 1083-1090.

MaY, C. P. (1999). Synchrony effects in cognition: The costs and a benefit. Psychonomic Bulletin \& Review, 6, 142-147.

MaY, C. P., \& Hasher, L. (1998). Synchrony effects in inhibitory control over thought and action. Journal of Experimental Psychology: Human Perception \& Performance, 24, 363-379.

May, C. P., Hasher, L, \& Foong, N. (2005). Implicit memory, age, and time of day: Paradoxical priming effects. Psychological Science, 16, 96-100.

Petros, T. V., Beckwith, B. E., \& Anderson, M. (1990). Individual differences in the effects of time of day and passage difficulty on prose memory in adults. British Journal of Psychology, 81, 63-72.

PfeIfFER, E. (1975). A short portable mental status questionnaire for the assessment of organic brain deficit in elderly patients. Journal of the American Geriatric Society, 23, 433-441.

Richardson-Klavehn, A., \& Gardiner, J. M. (1995). Retrieval volition and memorial awareness in stem completion: An empirical analysis. Psychological Research, 57, 166-178.

Roenneberg, T., Kuehnle, T., Pramstaller, P. P., Ricken, J., Havel, M., Guth, A., \& Merrow, M. (2004). A marker for the end of adolescence. Current Biology, 14, R1038-R1039.

RoßNAGEL, C. S. (2001). Revealing hidden covariation detection: Evidence for implicit abstraction at study. Journal of Experimental Psychology: Learning, Memory, \& Cognition, 27, 1276-1288.

Smith, C. S., Reilly, C., \& MidKIfF, K. (1989). Evaluation of three circadian rhythm questionnaires with suggestions for an improved measure of morningness. Journal of Applied Psychology, 74, 728-738.

Taillard, J., Philip, P., Chastang, J. F., \& Bioulac, B. (2004). Validation of Horne and Ostberg morningness-eveningness questionnaire in a middle-aged population of French workers. Journal of Biological Rhythms, 19, 76-86.

West, R., Murphy, K. J., Armilio, M. L., Craik, F. I. M., \& Stuss, D. T. (2002). Effects of time of day on age differences in working memory. Journals of Gerontology, 57B, P3-P10.

Wilson, D. E., \& HorTON, K. D. (2002). Comparing techniques for estimating automatic retrieval: Effects of retention interval. Psychonomic Bulletin \& Review, 9, 566-574.

WiNOCUR, G., \& HASHER, L. (2002). Circadian rhythms and memory in aged humans and animals. In L. R. Squire \& D. L. Schacter (Eds.), Neuropsychology of Memory (3rd ed., pp. 273-285). New York: Guilford.

Yoon, C. (1997). Age differences in consumers' processing strategies: An investigation of moderating influences. Journal of Consumer Research, 24, 329-342.

\section{NOTES}

1. On the basis of normative data, the majority of older adults are morning types and have a peak intellectual and physical period sometime in the morning, whereas a substantial group of young university students are evening types and have a peak time later in the day (Hasher et al., 2005; Roennenberg et al., 2004).

2. A recent study proposed new cutoff scores for morningness based on a sample of adults from 44 to 58 years of age (Taillard, Philip, Chastang, \& Bioulac, 2004). To be consistent with previous work (e.g., May et al., 2005), we did not change the cutoffs used here. As well, Taillard et al.'s "neither type" and "morning-type" people had the same peak performance period (8-10 a.m.) as that used in the present study.

(Manuscript received November 20, 2005; revision accepted for publication May 23, 2006.) 